\title{
EFETIVIDADE DA ESTABILIZAÇÃO SEGMENTAR VERTEBRAL E DE OUTRAS TÉCNICAS TERAPÊUTICAS EM DISFUNÇÕES DA COLUNA VERTEBRAL: REVISÃO SISTEMÁTICA
}

\author{
Evellyn Claudia Wietzikoski Lovato ${ }^{1}$ \\ Maria Helena Xavier Davila ${ }^{2}$ \\ Sandra Patrícia Ferreira Barros ${ }^{2}$ \\ Samantha Wietzikoski Sato ${ }^{3}$ \\ Francislaine Aparecida dos Reis Lívero \\ Irinéia Paulina Baretta ${ }^{1}$ \\ Maria de Fátima da Costa Almeida ${ }^{4}$ \\ Rúbia Márcia Benatti ${ }^{5}$
}

LOVATO, E. C. W.; DAVILA, M. H. X.; BARROS, S. P. F.; SATO, S. W.; LÍVERO, F. A. dos R.; BARETTA, I. P.; ALMEIDA, M. de F. da C.; BENATTI, R. M. Efetividade da estabilização segmentar vertebral e de outras técnicas terapêuticas em disfunções da coluna vertebral: revisão sistemática. Arq. Cienc. Saúde UNIPAR, Umuarama, v. 21, n. 3, p, 213-223, set./dez. 2017.

\begin{abstract}
RESUMO: As disfunções da coluna vertebral afetam uma grande parcela da população adulta em algum momento da vida, com predileção por adultos jovens em fase ativa. Por se tratar de uma patologia recorrente na população em geral torna-se necessário o desenvolvimento de estudos baseados em evidência que comprovem a efetividade de técnicas utilizadas no tratamento das disfunções da coluna vertebral. Assim, esta revisão sistematizada teve por objetivo abordar a efetividade da estabilização segmentar vertebral e de outras técnicas terapêuticas em indivíduos que apresentam disfunções da coluna vertebral. Para desenvolver esta revisão sistemática da literatura foram realizadas busca nas bases de dados PUBMED (National Library of Medicine and National Intiuttes of Health), PEDro (Physiotherapy Evidence Database) e SCIELO (Scientific Eletronic Library OnLine) no período de 2003 a 2014. Os descritores utilizados de forma isolada ou combinada foram: "segmental stabilization and manual therapy", "segmental stabilization", "manual therapy and spinal disorders". Foram incluídos no total 16 artigos que focaram indivíduos com disfunção da coluna vertebral lombar de causa não específica. A literatura analisada sugere que os exercícios de estabilização são positivos no tratamento de pacientes com disfunção da coluna lombar, entretanto são inconclusivos ao verificar se tais exercícios são superiores às técnicas de terapia manual ou a tratamentos convencionais como os exercícios gerais. PALAVRAS-CHAVE: Estabilização segmentar. Exercícios Terapêuticos. Disfunção da Coluna Vertebral. Terapia manual.
\end{abstract}

\section{SPINAL SEGMENTAL STABILIZATION EFFECTIVENESS AND OTHER THERAPEUTIC TECHNIQUES IN SPINE DYSFUNCTION: A SYSTEMATIC REVIEW}

\begin{abstract}
The spine dysfunctions affect a great portion of the adult population in some moment of the life, with predilection for young adults in activates phase. Due to be an appealing pathology becomes necessary the development of studies based in evidence that prove the effectiveness of techniques used in the treatment of the spine dysfunctions. Therefore, this systematized revision had for objective to approach the effectiveness of the vertebral segmentary stabilization and of other therapeutic techniques in individuals that present spine dysfunctions. To develop this systematic revision, it was accomplished search in the bases of data PUBMED (National Library of Medicine and National Intiuttes of Health), PeDRO (Physiotherapy Evidence Database) and SCIELO (Scientific Eletronic Library OnLine) in the period from 2003 to 2014. The used descritores in way isolated or combined were: "segmental stabilization and manual therapy", "segmental stabilization", "manual therapy and spinal disorders". It was included 16 articles that focused participants with lumbar spine dysfunction of cause no specific. The analyzed literature suggests that the exercises of stabilization are positive in the patients' treatment with back lumbar pain; however, they are inconclusive when verifying such exercises are superior to the techniques of manual therapy or to conventional treatments as the general exercises.
\end{abstract}

KEYWORDS: Manual therapy. Segmentary stabilization. Spine dysfunction. Therapeutic exercises.

\section{Introdução}

As disfunções da coluna vertebral afetam de $70 \%$ a $80 \%$ da população adulta em algum momento da vida (DEYO et al., 1991), com predileção por adultos jovens, em fase ativa (DE VITTA, 1996). De acordo com Organização Mundial da Saúde (OMS) estima-se que $80 \%$ da população mundial sofrerá algias da coluna lombar em algum momento de sua vida, sendo que $50 \%$ dos indivíduos já apresentaram este quadro álgico (GRIFFIN; HARMON; KENNEDY, 2012). Por se tratar de uma patologia recorrente na população em geral são necessários desenvolvimento de estudos baseados em evidência que comprovem a efetividade de técnicas utilizadas no tratamento das disfunções da coluna vertebral.

Evidências atuais mostram que o decréscimo da estabilidade do core predispõe a lesões e que, por isso, o treinamento apropriado pode reduzir a ocorrência destas lesões, entre elas as de origem cervical e lombar (PAVIN; GONÇALVES, 2010). O papel dos estabilizadores segmentares consiste em fornecer suporte e proteção às articulações por meio do controle fisiológico e translacional excessivo do movimento (FRANÇA et al., 2008). Com isso, esse tipo de treinamento

DOI: 10.25110 /arqsaude.v21i3.2017.6589

${ }^{1}$ Doutora em Farmacologia - Docente do Mestrado Profissional em Plantas Medicinais e Fitoterápicos na Atenção Básica da Universidade Paranaense UNIPAR, Umuarama, Paraná, Brasil.

${ }^{2}$ Fisioterapeuta Ortopédica e Traumatológica: Concentração em Terapia Manual e Postural pela Universidade Positivo, Curitiba, Paraná, Brasil.

${ }^{3}$ Mestre em Farmacologia. Docente da Universidade Paranaense - UNIPAR, Umuarama, Paraná, Brasil.

${ }^{4}$ Doutora em fisiologia pela Universidade Federal de São Paulo.

${ }^{5}$ Mestre em Ciências do Aparelho Locomotor pela Universidade Federal de São Paulo (UNIPFESP/EPM) Especialista em Fisioterapia Traumato-Ortopédica Funcional pela Universidade Positivo - Docente da Universidade Positivo e da Faculdade Evangélica do Paraná, Curitiba, Paraná, Brasil. 
pode atuar na prevenção de lesões, pois apesar de nem todas serem decorrentes da instabilidade dessa região, é consenso que os músculos do core influenciam toda a coluna vertebral (PAVIN; GONÇALVES, 2010).

Os exercícios de estabilização são essenciais para promover uma base para os movimentos de membros superiores e inferiores, para suportar cargas e para proteger a medula e as raízes nervosas (WILLARDSON; FONTANA; BRESSEL, 2009). Os programas de exercícios de estabilização visam melhorar a força, resistência e o controle motor dos músculos abdominais e lombares, com ênfase nos músculos profundos do tronco, principalmente transverso do abdômen e multífidos (VOLPATO et al., 2012). Além disso, é importante que seja enfatizado o treinamento de outros músculos paravertebrais, abdominais, bem como o diafragma e a musculatura pélvica (STANDAERT; WEINSTEIN; RUMPELTES, 2008), para que o tratamento seja eficaz na redução da dor (HEBERT, 2010; VOLPATO et al., 2012).

Outros estudos demonstram que tratamentos utilizando terapias manuais ou exercícios terapêuticos são capazes de promover efeitos benéficos em indivíduos com disfunções da coluna vertebral. Diversas são as técnicas fisioterapêuticas empregadas no tratamento destas disfunções, tais como as técnicas de Mulligan, que realizam mobilizações articulares associadas ao movimento ativo ou passivo (COLLINS; TEYS; VICENZINO, 2004; HALL et al., 2007), as técnicas de Maitland, que realizam mobilizações passivas fisiológicas e/ou acessórias e manipulações articulares (MAITLAND et al., 2007), técnicas quiropráxicas (ANDERSON-PEACOCK et al., 2005), técnicas osteopáticas (CHOWN et al., 2008) e técnicas de contração-relaxamento (MARCONDES; LODOVICHI; CERA, 2010; NUNES-JUNIOR; MONNERAT, 2012).

Assim, a necessidade de uma pesquisa voltada para o assunto em questão não se resume apenas na satisfação pelo ganho profissional ou pelo conhecimento adquirido, mas pelo fato de que a importância do tema é incalculável já que o problema atinge todas as classes sociais, sexos e diversas faixas etárias (em especial adultos jovens que estão em fase produtiva). Portanto, esta revisão sistemática provém informações sobre a prática clínica baseada em evidências, fornecendo dados da efetividade das técnicas analisadas visando resultados satisfatórios em tratamentos para a redução desse tipo de disfunção.

Desta forma, o objetivo deste estudo foi abordar a efetividade da estabilização segmentar vertebral e de técnicas de terapias manuais em indivíduos que apresentam disfunções da coluna vertebral, através de uma revisão sistematizada de literatura.

\section{Métodos}

Este é um estudo descritivo de revisão sistemática de literatura. A amostra foi composta por artigos selecionados conforme os critérios de inclusão e exclusão.

Para desenvolver esta revisão sistemática foi realizada uma busca nas bases de dados PUBMED (National Library of Medicine and National Institutes of Health), PEDro (Physiotherapy Evidence Database) e SCIELO (Scientific Eletronic Library OnLine) no período de 2003 a 2014. O idioma pré-estabelecido neste trabalho foi o inglês.
Foram excluídos estudos de revisão de literatura ou sistematizadas e/ou estudos originais com baixo grau de evidência científica (classificação Qualis Capes no mínimo B3) e/ou publicados previamente ao ano de 2003, além de estudos realizados em gestantes, idosos, ginastas ou em uso ginecológico (com enfoque para contração do períneo).

Os critérios de inclusão adotados foram artigos que relatavam os efeitos dos exercícios e a importância dos músculos profundos na manutenção da estabilização segmentar na reabilitação fisioterapêutica da coluna vertebral. Também foram considerados artigos que comparavam o efeito da estabilização segmentar com outras técnicas de tratamento envolvendo terapias manuais e/ou exercícios terapêuticos. Foram incluídos somente estudos originais ou ensaios clínicos que utilizavam alguma técnica para mensurar a melhora obtida pelo tratamento proposto, tais como as técnicas de biofeedback de pressão (stabilazer), eletromiografia, ultrassom e questionários e/ou inventários.

Três revisores independentes realizaram a busca dos artigos conforme os critérios de seleção nas bases de dados com os seguintes descritores (isolados ou combinados): "segmental stabilization and manual therapy", "segmental stabilization", "manual therapy and spinal disorders".

Após a busca sistemática dos artigos foi realizada a leitura crítica e selecionados aqueles que cumpriram os critérios de inclusão e que ao mesmo tempo demonstraram-se relevantes para o desenvolvimento do trabalho. Posteriormente à análise do material coletado para a pesquisa, foi realizado um resumo em forma de tabelas.

Os dados foram tabulados nas seguintes variáveis: autores/ano, sujeitos do estudo, método (critérios de avaliação), intervenções e resultados. A organização e a análise dos dados serviram como base para a realização desta revisão de literatura na qual foi desenvolvida uma discussão fundamentada em resultados científicos.

\section{Resultados e Discussão}

Para a realização deste estudo sistematizado foram consultadas as bases de dados eletrônicas: PubMed, PEDro e Scielo, dividindo esta tarefa em quatro fases conforme diagramado na figura 1. Como estratégia de pesquisa utilizou-se os mesmos descritores em todos os bancos de dados, conforme descrito na metodologia.

Em uma primeira análise foram encontrados no total 14 artigos, sendo 11 no PubMed e 3 no PEDro (fase 1). Nenhum artigo foi encontrado na base de dados Scielo utilizando os descritores pré-estabelecidos. Após a leitura crítica dos resumos foram constatados que somente 11 artigos encontravam-se de acordo com os critérios de inclusão (fase 2). $\mathrm{Na}$ sequência, foram avaliadas as referências bibliográficas dos 11 artigos encontrados e a partir disto processou-se uma nova busca nas bases de dados (fase 3), resultando no achado de 5 artigos. Após a análise dos resumos destes artigos foram verificados os critérios de inclusão e exclusão, totalizando ao final da busca sistematizada 16 artigos científicos classificados para o estudo (fase 4). Portanto, no total foram avaliados integralmente 16 artigos, publicados entre 2003 e 2014. 
Figura 1: Diagrama dos procedimentos adotados durante a busca sistematizada dos artigos nas bases de dados eletrônicas PubMed, PEDro e Scielo.

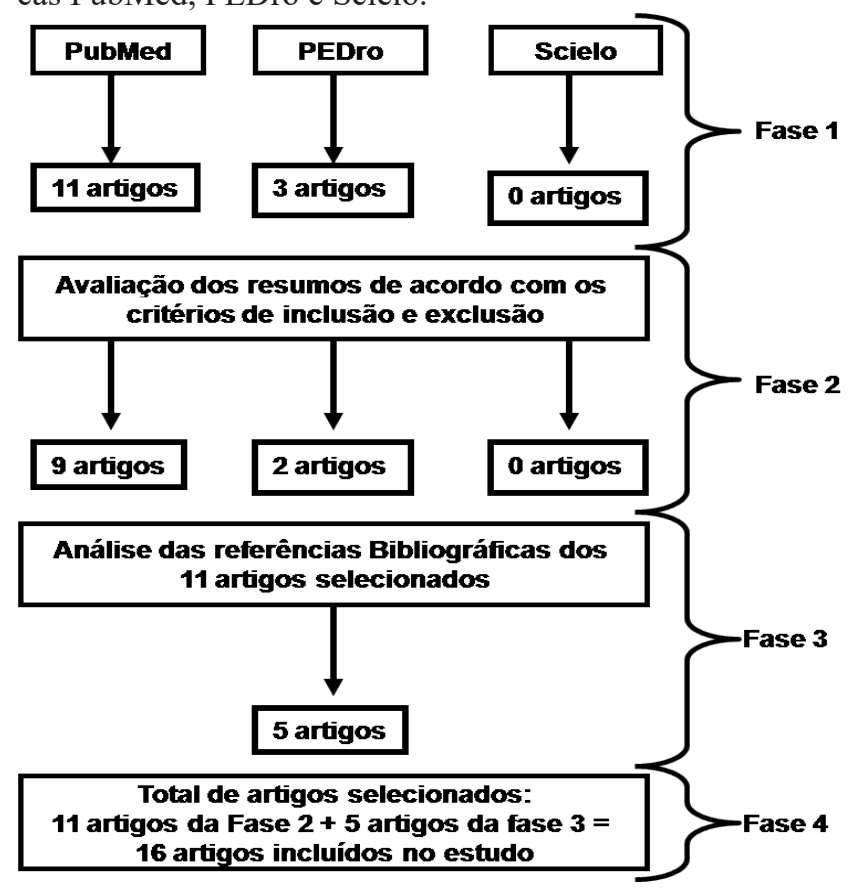

\section{Caracterização dos artigos incluídos}

Todos os estudos objetivaram comparar a aplicação da técnica de estabilização central com algum outro método terapêutico envolvendo terapia manual ou exercícios terapêuticos em disfunções da coluna vertebral.

Os artigos, em sua maioria, foram publicados nos anos de 2005 e 2010, porém, pelo menos um estudo por ano foi encontrado nos demais anos pesquisados, conforme demonstrado na Tabela 1, que descreve as características referentes ao tamanho da amostra, faixa etária e gênero da população estudada.

Tabela 1: Características das publicações selecionadas para a revisão sistematizada sobre o emprego de técnicas de estabilização segmentar vertebral e de outras técnicas terapêuticas em indivíduos que apresentam disfunções da coluna vertebral, no que se refere ao tamanho da amostra, faixa etária e gênero.

\begin{tabular}{|c|c|c|c|}
\hline AUTOR/ANO & AMOSTRA & $\begin{array}{c}\text { FAIXA } \\
\text { ETÁRIA }\end{array}$ & GÊNERO \\
\hline Ahmed, Rehman e Sibtain, 2014 & 40 & NI & $\mathrm{M} / \mathrm{F}$ \\
\hline Aluko, DeSouza e Peacock, 2013 & 33 & Media 36 & $\mathrm{M} / \mathrm{F}$ \\
\hline França et al., 2012 & 30 & Média 42 & NI informado \\
\hline Saner et al., 2011 & 106 & 18 a 75 & NI informado \\
\hline Kumar, 2011 & 18 & Média 22 & $\mathrm{M} / \mathrm{F}$ \\
\hline Unsgaard-Tondel et al., 2010 & 109 & 19 a 60 & $\mathrm{M} / \mathrm{F}$ \\
\hline Muthukrishnan et al., 2010 & 65 & 18 a 55 & NI informado \\
\hline Ferreira et al., 2010 & 34 & 18 a 80 & $\mathrm{~F}$ \\
\hline França et al., 2010 & 30 & Média 42 & NI informado \\
\hline Ewert et al., 2009 & 169 & 18 a 65 & $\mathrm{~F}$ \\
\hline Carpes, Reinehr e Mota, 2008 & 106 & 18 a 75 & $\mathrm{~F}$ \\
\hline Stevens et al., 2007 & 30 & 19 a 23 & $\mathrm{M} / \mathrm{F}$ \\
\hline Fritz, Whitman e Childs, 2005 & 131 & 19 a 59 & $\mathrm{M} / \mathrm{F}$ \\
\hline Hicks et al., 2005 & 54 & Media 42,4 & $\mathrm{M} / \mathrm{F}$ \\
\hline Koumantakis, Watson e Oldham, 2005 & 55 & Media 39,2 & $\mathrm{NI}$ informado \\
\hline Rasmussen-Barr, Nilsson-Wikmarn e Arvidsson, 2003 & 47 & 18 a 60 & $\mathrm{M} / \mathrm{F}$ \\
\hline
\end{tabular}

F- Feminino. NI- Não informado. M-Masculino.
Nos estudos avaliados observou-se que o tamanho da amostra variou de 18 a 169 participantes. O dimensionamento da amostra é um processo matemático de decisão, no qual se define quantos indivíduos devem ser estudados (LAST, 1996). O tamanho amostral é importante por questões éticas e econômicas (MacFARLANE, 2003). Um estudo utilizando amostra pequena pode não produzir resultados úteis, expondo os seus participantes a riscos desnecessários, enquanto uma amostra de tamanho excessivo usa mais fonte de recursos do que é necessário, além de expor um excessivo número de indivíduos aos riscos porventura existentes (NORMANDO; ALMEIDA; QUINTÃO, 2011).

Nos artigos avaliados, notou-se que cinco trabalhos definiram a amostragem por conveniência. Segundo Gil (1999), a amostragem por conveniência constitui o menos rigoroso de todos os tipos de amostragem; por isso, é destituído de qualquer rigor estatístico. Esse tipo de amostragem é aplicado em estudos exploratórios ou qualitativos, nos quais não é requerido elevado nível de precisão. Nenhum dos trabalhos avaliados citou cálculo de amostragem, entretanto a maioria utilizou a randomização para alocar os grupos experimentais. Em relação à faixa etária, observou-se que os participantes apresentavam idade entre 18 a 80 anos (média de 31 anos), sendo, portanto, considerada a população de maior risco em apresentar disfunções na coluna vertebral. Estudos prévios evidenciam que as disfunções da coluna vertebral, em especial as lombalgias, apresentam alta incidência em adultos jovens e em idosos (VITTA, 1996).

Ao verificar o gênero predominante na população estudada, foi evidenciado que apenas oito dos dezesseis artigos se referiram a análise de ambos os sexos, três utilizaram somente participantes do sexo feminino e cinco não mencionam qual foi o sexo analisado, apesar de subentender a participação de ambos os sexos.

\section{Métodos de avaliação empregados nos artigos incluídos}

As causas de disfunções da coluna vertebral são processos degenerativos, inflamatórios, alterações congênitas e mecânico posturais. Estas últimas são responsáveis por grande parte das dores. Nelas ocorre um desequilíbrio entre a carga funcional (esforço requerido para atividades do trabalho e da vida diária) e a capacidade funcional (potencial para a execução) (DEYO et al., 1991). Os trabalhos incluídos no presente estudo, definidos a partir das palavras-chave estabelecidas como critérios de inclusão, focaram indivíduos com disfunção da coluna vertebral lombar, no qual apresentavam causas não específicas.

As lombalgias de causa não específica podem ser definidas como aquelas em que a dor lombar presente ocorre sem o conhecimento de patologia subjacente (KRISMER; VAN TULDER, 2007). Esta forma de disfunção da coluna vertebral apresenta grande prevalência na população em geral, ocasionando encargos financeiros à sociedade principalmente devido aos custos indiretos relacionados aos pedidos de afastamentos do trabalho (KRISMER; VAN TULDER, 2007).

Portanto, tendo em vista a alta prevalência mencionada, foi observado nesta revisão sistematizada que a busca criteriosa resultou em trabalhos científicos que estudavam indivíduos com lombalgia de causa não específica para ve- 
rificar a eficácia da aplicação de exercícios de estabilização central e outras técnicas terapêuticas.

Em relação aos métodos utilizados para avaliar a eficácia das intervenções terapêuticas realizadas nos trabalhos, verificou-se o emprego de dezoito diferentes métodos, conforme descrito na Tabela 2.

Tabela 2: Métodos de avaliação utilizados pelos autores dos artigos científicos selecionadas para a revisão sistematizada.

\section{MÉTODOS DE AVALIAÇÃO}

Escala Visual Analógica da Dor (EVA)

Escala Visual Analogica da Dor (EVA)

Escala de Incapacidade de Oswestry

Questionário de Incapacidade de Roland Morris

\begin{tabular}{l|}
\hline Questionário de McGill \\
\hline Monitor de Medição Lombar (LMM) \\
\hline Aparelho de Biofeedback de Pressão \\
\hline Algometro de Pressão \\
\hline Escala Funcional Específica \\
\hline $\begin{array}{l}\text { Escala de Dor Crônica Graduada } \\
\text { (CPQ) }\end{array}$
\end{tabular}

\begin{tabular}{l|l}
\hline Escala Graduada Joint Play & \\
\hline Escala Numérica de Frequência da & \\
Dor & \\
\hline Questionário de Condicionamento de & \\
Medo (FABQ) & \\
\hline
\end{tabular}

Ultrassonografia
Inventário Multidimensional da Dor
(MPI-D)

\begin{tabular}{l|l}
\hline Eletromiografia (EMG) & Stevens et al., 2007 \\
\hline Escala de Locus & Koumantakis, Watson e Oldham, 2005 \\
\hline Questionário Dor auto-Referida & Koumantakis, Watson e Oldham, 2005 \\
\hline Tampa Scale de Kinesiophobia & Koumantakis, Watson e Oldham, 2005 \\
\hline
\end{tabular}

É possível observar que os instrumentos de avaliação mais utilizados foram: Escala Visual Analógica da Dor (EVA) (ALUKO; KUMAR, 2001; HICKS et al., 2005; KOUMANTAKIS; WATSON; OLDHAM, 2005; FERREIRA et al., 2010; FRANÇA, 2010; FRANÇA, 2012; De SOUZA; PEACOCK, 2013; AHMED; SHAKIL-UR-REHMAN; SIBTAIN, 2014;), Escala de Incapacidade de Oswestry (FRITZ; RASMUSSEN-BARR; NILSSON-WIKMAR; ARVIDSSON, 2003; WHITMAN; CHILDS, 2005; HICKS, 2005; FRANÇA et al., 2010; UNSGAARD-TONDEL et al., 2010; FRANÇA, 2012; AHMED; SHAKIL-UR-REHMAN; SIBTAIN, 2014), Questionário de Incapacidade de Roland Morris (KOUMANTAKIS; WATSON; OLDHAM, 2005; CARPES; REINEHR; MOTA, 2008; MUTHUKRISHNAN et al., 2010; SANER et al., 2011; ALUKO; De SOUZA; PEACOCK, 2013), Questionário de McGill (KOUMANTAKIS; WATSON; OLDHAM, 2005; FRANÇA et al., 2010; 2012) e o Questionário de Condicionamento de medo (FABQ) (HICKS et al., 2005; MUTHUKRISHNAN et al., 2010; UNSGAARD-TONDEL et al., 2010).

Como o principal sintoma relacionado à disfunção da coluna lombar refere-se a dor, muitos estudos utilizaram

Aluko, DeSouza e Peacock, 2013

França et al., 2012; França et al., 2010

Saner et al., 2011

Unsgaard-Tondel et al., 2010

Ferreira et al., 2010

\section{AUTORES/ANO}

Ahmed, Rehman e Sibtain, 2014; Aluko, DeSouza e Peacock, 2013; França et 2005; Koumantakis, Watson e Oldham, 2005

Ahmed, Rehman e Sibtain, 2014; França et al., 2012; Unsgaard-Tondel et al., Rasmussen-Barr, Nilsson-Wikmarn e Arvidsson, 2003

Aluko, DeSouza e Peacock, 2013; Saner et al., 2011; Muthukrishnan et al., 2010, Carpes, Reinehr e Mota, 2008; Koumantakis, Watson e Oldham, 2005

Saner et al., 2011; Muthukrishnan et al., 2010; Carpes, Reinehr e Mota, 2008

Muthukrishnan et al., 2010; Unsgaard-Tondel et al., 2010; Hicks et al., 2005

a EVA e/ou o Questionário de McGill para verificar a eficácia terapêutica dos exercícios propostos. A EVA é constituída por uma linha de $10 \mathrm{~cm}$ que tem, em geral, como extremos as frases “ausência de dor e dor insuportável". Apesar de ser um instrumento de fácil aplicação, muitas vezes os indivíduos, principalmente crianças e idosos apresentam dificuldades em utilizá-la devido à abstração necessária para sua compreensão (GIFT, 1989). Já o Questionário de McGill, é uma escala que mensura as diferentes qualidades da dor que possam ser analisadas estatisticamente (MELZACK, 1987).

A primeira parte do questionário contém um esboço do corpo humano usado para fazer a localização espacial e em profundidade da dor referida pelo paciente. A segunda parte busca coletar informações sobre as propriedades temporais da dor (contínuas, ritmadas, momentâneas), as circunstâncias em que os sintomas dolorosos começaram a ser percebidos e as intervenções analgésicas que estão sendo ou que já foram usadas para minimizá-la. A terceira parte ajuda o paciente a relatar as qualidades específicas de suas dores. Ela prevê um espaço para a queixa espontânea da dor, mas é formada, principalmente, por um conjunto de 68 palavras que descrevem diversas qualidades das experiências doloro- 
sas em geral e que são escolhidas pelos pacientes para caracterizar as suas dores em particular. A quarta parte busca avaliar a Intensidade da Dor Presente. Trata-se de uma escala âncora alfa - numérica que varia de 1 a 5, associada com as seguintes palavras: (1) fraca; (2) moderada; (3) forte; (4) violenta e (5) insuportável. A escolha dos descritores para nomear a dor vai depender da experiência prévia do indivíduo, do estresse emocional gerado pela condição de saúde, pelas peculiaridades e especificidade do indivíduo, gerando assim a qualidade de sua dor (SANTOS et al., 2006).

Em pacientes com disfunção da coluna vertebral de causa não específica, os exames médicos complementares são muitas vezes normais, não ajudando a quantificação da sua incapacidade funcional (MONTEIRO et al., 2010). Assim, para comparar e avaliar o tratamento e a evolução dos indivíduos durante os experimentos foi necessário a aplicação de instrumentos que quantifiquem esta limitação funcional. Neste sentido, a Escala de Incapacidade de Oswestry foi concebida para avaliar como a dor afeta várias atividades da vida diária e atribui uma pontuação percentual relacionado ao nível de função (FAIRBANK; PYNSENT, 2006). Trata-se de um questionário autoaplicável, dividido em dez seções que investiga a intensidade da dor, cuidados pessoais, pesos, andar, sentar, de pé, sono, vida sexual, vida social e viagens. A cada item atribui-se um valor de 0 a 5 pontos, sendo interpretados percentualmente e classificados em incapacidade mínima ( $0 \%$ a $20 \%)$, incapacidade moderada $(21 \%$ a $40 \%)$, incapacidade intensa (41\% a $60 \%)$, aleijado (61\% a $80 \%)$ e inválido ( $81 \%$ a $100 \%)$. O questionário apresenta ainda interpretação de resultados no pós-operatório.

Já o Questionário de Incapacidade de Roland Morris foi utilizado para avaliar a incapacidade funcional de indivíduos com lombalgia nas suas atividades diárias ${ }^{44}$. O questionário é constituído por 24 perguntas de auto-resposta que os indivíduos preenchem em menos de cinco minutos (ROLAND; MORRIS, 1983; ROUWER, 2004).

O Questionário de Condicionamento de Medo (FABQ) possui 2 sub-escalas que medem crenças e medo dos indivíduos em relação ao trabalho (sete itens) e em relação a atividade física (quatro itens) (WADDELL et al., 1993).

Outros métodos também foram utilizados em um menor número de artigos, tais como a eletromiografia (STEVENS et al., 2007), ultrassonografia (FERREIRA et al, 2010), biofeedback de pressão (FRANÇA et al., 2010; 2012) e o algômetro de pressão (KUMAR, 2011).

É possível observar que os artigos analisados basearam seus resultados principalmente na resposta subjetiva de questionários, escalas e inventários aplicados antes e após as intervenções terapêuticas. Isto evidencia a dificuldade metodológica existente em se obter dados que comprovem efetivamente os efeitos dos exercícios terapêuticos e ou de estabilização central, pois conforme já mencionado anteriormente, os exames complementares de imagem de indivíduos com lombalgia, de causa não específica, muitas vezes são normais (MONTEIRO et al., 2010) e não auxiliam a quantificação do resultado da intervenção adotada.

\section{Intervenções terapêuticas realizadas nos artigos incluídos na revisão sistematizada}

Na tabela 3 encontram-se sumarizados as intervenções terapêuticas realizadas nos artigos analisados. São descritos os grupos experimentais, os procedimentos adotados e o tempo e intervalo de duração dos procedimentos.

Tabela 3: Intervenções terapêuticas realizadas pelos autores das publicações selecionadas para a revisão sistematizada.

\begin{tabular}{|c|c|c|c|}
\hline AUTOR /ANO & GRUPOS & PROCEDIMENTOS & INTERVALO \\
\hline $\begin{array}{l}\text { Ahmed, Rehman e } \\
\quad \text { Sibtain, } 2014\end{array}$ & $\begin{array}{l}\text { Grupo A: ML + EC } \\
\text { Grupo B: ML }\end{array}$ & $\begin{array}{l}\text { ML: movimento central antero-posterior realizado } \\
\text { nas vértebras de T12 a L5. } \\
\text { EC: exercícios de estabilização central. }\end{array}$ & $\begin{array}{l}1 \text { intervenção de } 45 \text { min } 4 \text { vezes } \\
\text { por semana durante } 6 \text { semanas }\end{array}$ \\
\hline $\begin{array}{l}\text { Aluko, DeSouza e } \\
\text { Peacock, } 2013\end{array}$ & $\begin{array}{l}\text { Grupo Exercício Regular } \\
\text { (ER) } \\
\text { Grupo Intervenção (GI) }\end{array}$ & $\begin{array}{l}\text { ER: Exercícios clássicos de core e de } \\
\text { fortalecimento global com mínimo de instrução. } \\
\text { GI: Mesmo protocolo com instruções adicionais. }\end{array}$ & $\begin{array}{l}\text { Procedimentos realizados por } 6 \\
\text { semanas }\end{array}$ \\
\hline França et al., 2012 & $\begin{array}{l}\text { Grupo EC } \\
\text { Grupo Isometria (IT) }\end{array}$ & $\begin{array}{l}\text { EC: Exercícios focando multífidos e transverso } \\
\text { abdominal. } \\
\text { IT: Exercícios focando isometria do músculo } \\
\text { eretor da espinha e tríceps sural. }\end{array}$ & $\begin{array}{l}2 \text { intervenções de } 30 \text { min por } \\
\text { semana durante } 6 \text { semanas }\end{array}$ \\
\hline Saner et al., 2011 & $\begin{array}{l}\text { Grupo EC } \\
\text { Grupo Exercícios Gerais } \\
\text { (EG) }\end{array}$ & $\begin{array}{l}\text { EC: Exercícios de controle do movimento } \\
\text { especíicos instruídos. } \\
\text { EG: protocolo de exercícios focando flexibilidade } \\
\text { e endurance. }\end{array}$ & Realizados 9 a 18 intervenções. \\
\hline Kumar, 2011 & $\begin{array}{l}\text { Grupo Placebo } \\
\text { Grupo Experimental }\end{array}$ & $\begin{array}{l}\text { Placebo: manter travesseiro entre as pernas em } \\
\text { posição prono durante } 15 \text { min, sob supervisão. } \\
\text { Experimental: exercícios de EC na posição de } \\
\text { gatas. }\end{array}$ & $\begin{array}{l}\text { Intervenção cruzada }- \text { os grupos } \\
\text { realizaram os procedimentos } \\
\text { e após intervalo de } 24 \text { horas, } \\
\text { inverteram os grupos. }\end{array}$ \\
\hline $\begin{array}{l}\text { Unsgaard-Tondel et } \\
\text { al., } 2010\end{array}$ & $\begin{array}{l}\text { Grupo ECM } \\
\text { Grupo EC } \\
\text { Grupo EG }\end{array}$ & $\begin{array}{l}\text { ECM: Exercícios de controle motor de baixa } \\
\text { carga guiado por ultrassom e instruídos } \\
\text { individualmente. } \\
\text { EC: Exercícios de controle motor de alta carga } \\
\text { guiado por ultrassom e instruídos individualmente. } \\
\text { EG: Exercícios gerais sem instrução. }\end{array}$ & $\begin{array}{l}1 \text { intervenção por semana } \\
\text { durante } 8 \text { semanas }\end{array}$ \\
\hline
\end{tabular}




\begin{tabular}{|c|c|c|c|}
\hline $\begin{array}{l}\text { Muthukrishnan et } \\
\text { al., } 2010\end{array}$ & $\begin{array}{l}\text { Grupo convencional } \\
\text { Grupo EC } \\
\text { Grupo controle }\end{array}$ & $\begin{array}{l}\text { Convencional: exercícios de fisioterapia } \\
\text { convencional. } \\
\text { EC: exercícios de estabilização central. } \\
\text { Grupo controle: nenhuma atividade. }\end{array}$ & $\begin{array}{l}1 \text { intervenção de } 45 \text { min } 4 \text { vezes } \\
\text { por semana durante } 8 \text { semanas }\end{array}$ \\
\hline Ferreira et al., 2010 & $\begin{array}{l}\text { Grupo } 1 \\
\text { Grupo } 2 \\
\text { Grupo } 3\end{array}$ & $\begin{array}{l}\text { 1: EC focando o movimento lombopélvico e a } \\
\text { estabilidade. } \\
\text { 2: Exercícios gerais seguindo protocolo de Klaber } \\
\text { Moffet e Frost. } \\
\text { 3: Terapia manipulativa em coluna e pelve, sem } \\
\text { thrust. }\end{array}$ & $\begin{array}{l}12 \text { intervenções durante } 8 \\
\text { semanas. }\end{array}$ \\
\hline França et al., 2010 & $\begin{array}{l}\text { Grupo EC } \\
\text { Grupo Fortalecimento } \\
\text { Superficial (FS) }\end{array}$ & $\begin{array}{l}\text { EC: Exercícios focando multífidos e transverso } \\
\text { abdominal. } \\
\text { FS: Exercícios focando reto abdominal, oblíquos } \\
\text { internos e externos e eretor. }\end{array}$ & $\begin{array}{l}2 \text { intervenções de } 30 \text { min por } \\
\text { semana durante } 6 \text { semanas }\end{array}$ \\
\hline Ewert et al., 2009 & $\begin{array}{l}\text { Grupo EG } \\
\text { Grupo Programa } \\
\text { Multimodal (PM) }\end{array}$ & $\begin{array}{l}\text { EG: Exercícios gerais seguindo protocolo de } \\
\text { Klaber Moffet e Frost } \\
\text { PM: combinação de EC, exercícios domiciliares, } \\
\text { exercícios ergonômicos e atividades psicológicas }\end{array}$ & $\begin{array}{l}\text { EG: } 11 \text { intervenções de } 1 \text { hora. } \\
\text { PM: } 18 \text { intervenções, sendo } 17 \\
\text { de } 1,75 \text { h e } 1 \text { de } 45 \text { min. }\end{array}$ \\
\hline $\begin{array}{l}\text { Carpes, Reinehr e } \\
\text { Mota, } 2008\end{array}$ & Grupo único & $\begin{array}{l}\text { Realizado treinamento com exercícios de } \\
\text { fortalecimento muscular e de estabilização } \\
\text { central. }\end{array}$ & $\begin{array}{l}20 \text { intervenções de } 50 \text { min } \\
\text { durante } 7 \text { semanas. }\end{array}$ \\
\hline Stevens et al., 2007 & \begin{tabular}{|l|} 
Grupo 1 \\
Grupo 2 \\
\end{tabular} & $\begin{array}{l}\text { 1: EC em posição supino em ponte } \\
\text { 2: EC em posição gatas }\end{array}$ & $\begin{array}{l}\text { Antes e após treino específico de } \\
\text { estabilização central. }\end{array}$ \\
\hline $\begin{array}{l}\text { Fritz, Whitman e } \\
\text { Childs, } 2005\end{array}$ & $\begin{array}{l}\text { Grupo } 1 \\
\text { Grupo } 2 \\
\end{array}$ & $\begin{array}{l}\text { 1: Manipulação combinado a exercícios gerais } \\
2 \text { EC }\end{array}$ & 5 intervenções \\
\hline Hicks et al., 2005 & $\begin{array}{l}\text { Grupo } 1 \\
\text { Grupo } 2\end{array}$ & $\begin{array}{l}\text { 1: EG combinados com EC dos músculos } \\
\text { específicos do tronco. } \\
\text { 2: EG }\end{array}$ & $\begin{array}{l}2 \text { intervenções por semana } \\
\text { durante } 8 \text { semanas }\end{array}$ \\
\hline $\begin{array}{l}\text { Koumantakis, } \\
\text { Watson e Oldham, } \\
2005\end{array}$ & $\begin{array}{l}\text { Grupo } 1 \\
\text { Grupo2 }\end{array}$ & $\begin{array}{l}\text { 1: EC } \\
2: \mathrm{EG}\end{array}$ & $\begin{array}{l}\text { Intervenções realizadas por } 8 \\
\text { semanas. }\end{array}$ \\
\hline $\begin{array}{l}\text { Rasmussen-Barr, } \\
\text { Nilsson-Wikmarn e } \\
\text { Arvidsson, } 2003 \\
\end{array}$ & $\begin{array}{l}\text { Grupo EC } \\
\text { Grupo Terapia Manual } \\
\text { (TM) }\end{array}$ & $\begin{array}{l}\text { EC: exercícios de estabilização central } \\
\text { MT: técnicas de terapia manual }\end{array}$ & $\begin{array}{l}1 \text { intervenção de } 45 \text { min por } \\
\text { semana durante } 6 \text { semanas }\end{array}$ \\
\hline
\end{tabular}

ML: Mobilização Lombar, EC: Estabilização Central, ECM: Exercícios de Controle Motor de Baixa Carga.

É possível observar que quatro artigos (HICKS, 2005; CARPES; REINEHR; MOTA, 2008; ALUKO; EWERT et al., 2009; De SOUZA; PEACOCK, 2013) combinaram os exercícios de estabilização central com exercícios gerais de fortalecimento e compararam as técnicas combinadas com exercícios de estabilização central isolado. Cinco artigos (KOUMANTAKIS; WATSON; OLDHAM, 2005; FRANÇA et al., 2010; MUTHUKRISHNAN et al., 2010; UNSGAARD-TONDEL et al., 2010; SANER, et al., 2011) estudaram os efeitos da estabilização central comparado a um programa de exercícios gerais. Dois artigos estudaram somente os efeitos da estabilização central, no qual um realizou a comparação com o grupo placebo (UNSGAARD-TONDEL et al., 2010) e o outro comparou posições diferentes de treinos específicos de core (STEVENS et al., 2007). Três artigos (RASMUSSEN-BARR; NILSSON-WIKMAR; ARVIDSSON FRITZ, 2003; WHITMAN; CHILDS, 2005; AHMED; SHAKIL-UR-REHMAN; SIBTAIN, 2014) compararam os efeitos da estabilização central com técnicas de terapias manuais manipulativas. Um artigo comparou os efeitos da estabilização central, exercícios gerais e técnicas manipulativas (FERREIRA et al., 2010). E um artigo estudou os efeitos da isometria postural (FRANÇA et al., 2012) comparado a estabilização central.

As mobilizações articulares suaves de pouca ampli- tude e várias formas de mobilização dos tecidos moles vêm sendo utilizadas, há muito tempo, para o alívio da dor. Essas técnicas diminuem a dor mediante a modificação da dor reflexa, a inibição do espasmo muscular e a redução do edema (KALTENBORN, 2001).

Através de mobilização articular ocorre estimulação dos mecanoreceptores articulares beneficiando pacientes com disfunção mecânica da coluna vertebral. Acredita-se que esses receptores alteram o ciclo espasmo dor através da inibição pré-sináptica das fibras nociceptivas e a inibição de músculos hipertônicos (WILSON, 2001).

$\mathrm{O}$ alivio da dor observado através da terapia manual decorre da ativação dos sistemas inibitórios de dor descendentes que se projetam do mesencéfalo, conforme estudo realizado por Vicenzino, Collins e Wright (1998).

Recentemente têm se discutido os benefícios de técnicas de estabilização central em indivíduos com dores na coluna vertebral. Evidências atuais mostram que o decréscimo da estabilidade de core predispõe a lesões e que, por isso, o treinamento apropriado pode reduzir a ocorrência destas lesões, entre elas as de origem cervical e lombar (PAVIN; GONÇALVES, 2010). Assim, esse tipo de treinamento pode atuar na prevenção de lesões, pois apesar de nem todas serem decorrentes de instabilidade dessa região, é consenso que os músculos de core influenciam toda a coluna vertebral. 
A estabilidade da coluna depende do sistema muscular que compensa a instabilidade aumentando a resistência da coluna lombar. Tanto os músculos do tronco como os da pelve têm um papel na estabilização das articulações da coluna (STANMORE, 2008).

A estabilidade vertebral depende da integração entre 3 elementos: o sistema passivo que consiste dos corpos vertebrais, articulações facetarias, cápsulas articulares, ligamentos espinhais, discos intervertebrais que participam da estabilização por meio das propriedades viscoelásticas; o sistema ativo, constituído dos músculos espinhais e seus tendões; e o controle neural que recebe informações dos sistemas passivo e ativo, por meio dos receptores, e tem o papel de captar as alterações de equilíbrio e determinar os ajustes específicos, por meio da musculatura da coluna, restaurando a estabilidade (PANJABI, 2003). Quando um desses sistemas falha os outros dois se reorganizam para dar continuidade a homeostase. Porém, muitas vezes, essa reorganização é inadequada sobrecarregando os subsistemas, promovendo uma cronicidade da disfunção vertebral (PANJABI, 2003; STANMORE, 2008).

Os exercícios de estabilização são essenciais para promover uma base para os movimentos de membros superiores e inferiores, para suportar cargas e para proteger a medula e as raízes nervosas (WILLARDSON; FONTANA; BRESSEL, 2009). Os programas de exercícios de estabili- zação visam melhorar a força, resistência e o controle motor dos músculos abdominais e lombares, com ênfase nos músculos profundos do tronco, principalmente transverso do abdômen e multífidos (VOLPATO et al., 2012). Além disso, é importante que seja enfatizado o treinamento de outros músculos paravertebrais, abdominais, bem como o diafragma e a musculatura pélvica (STANDAERT; WEINSTEIN; RUMPELTES, 2008), para que o tratamento seja eficaz na redução da dor (HERBERT et al., 2010; VOLPATO et al., 2012). Desta forma é fato que os exercícios de estabilização central combinados a outras técnicas terapêuticas são capazes de promover benefícios, principalmente no que se refere a dor e a instabilidade de indivíduos com lombalgia de causa não específica.

\section{Resultados obtidos nos artigos incluídos na revisão siste- matizada}

Esta revisão sistematizada demonstrou que existem evidências sobre a eficácia dos exercícios de estabilização segmentar na melhora da dor em disfunções da coluna vertebral, quando estes são realizados isoladamente, ou quando são associados a outras técnicas terapêuticas. Porém, não foi possível evidenciar se os exercícios de estabilização são superiores às técnicas de terapia manual ou a outros tipos de intervenções convencionais, conforme descrito na Tabela 4.

Tabela 4: Resultados obtidos pelos autores das publicações selecionadas para a revisão sistematizada.

\begin{tabular}{|c|c|}
\hline AUTORES/ANO & RESULTADOS \\
\hline $\begin{array}{l}\text { Ahmed, Rehman e } \\
\text { Sibtain, } 2014\end{array}$ & $\begin{array}{l}\text { Exercícios de estabilização central associado a mobilização lombar reduz a dor e melhora a } \\
\text { incapacidade física dos indivíduos com lombalgia. }\end{array}$ \\
\hline $\begin{array}{l}\text { Aluko, DeSouza e } \\
\text { Peacock, } 2013\end{array}$ & $\begin{array}{l}\text { Os resultados foram inconclusivos sobre os efeitos benéficos dos exercícios específicos } \\
\text { de estabilização central na redução da dor aguda da coluna lombar, pois ambos os grupos } \\
\text { obtiveram resultados semelhantes. }\end{array}$ \\
\hline França et al., 2012 & $\begin{array}{l}\text { Ambos os tratamentos foram efetivos na redução da dor e na melhora da incapacidade da } \\
\text { coluna lombar, sendo que o grupo estabilização central apresentou escores mais elevados em } \\
\text { todas as variáveis quando comparado ao grupo submetido a exercícios de isometria postural. }\end{array}$ \\
\hline Saner et al., 2011 & Estudo ainda não finalizado. \\
\hline Kumar, 2011 & $\begin{array}{l}\text { O exercício de estabilização central foi mais efetivo comparado ao placebo na redução da } \\
\text { instabilidade segmentar lombar sintomática nas variáveis avaliadas. }\end{array}$ \\
\hline $\begin{array}{l}\text { Unsgaard-Tondel et al., } \\
2010\end{array}$ & $\begin{array}{l}\text { Não houve diferença significativa entre os grupos no que se refere a dor ou qualquer outra } \\
\text { medida avaliada no pós-tratamento imediato. Após } 1 \text { ano, foi observado diferenças entre os } \\
\text { grupos, sendo observado que os exercícios instruídos (grupos EMC e EC) foram superiores aos } \\
\text { exercícios gerais (grupo EG) para redução da dor. }\end{array}$ \\
\hline $\begin{array}{l}\text { Muthukrishnan et al., } \\
2010\end{array}$ & $\begin{array}{l}\text { O grupo submetido aos exercícios de estabilização central demonstrou melhora significativa } \\
\text { após a intervenção na graduação da força indicando mudanças nas vias de transferências de } \\
\text { carga comparado ao grupo controle. }\end{array}$ \\
\hline Ferreira et al., 2010 & $\begin{array}{l}\text { O grupo submetido a exercícios de estabilização central apresentou melhora no recrutamento } \\
\text { do transverso abdominal comparado aos grupos de exercício geral e terapias manipulativas. }\end{array}$ \\
\hline França et al., 2010 & $\begin{array}{l}\text { Ambos os tratamentos foram efetivos na redução da dor e na melhora da incapacidade da coluna } \\
\text { lombar, entretanto o grupo estabilização central apresentou ganhos significativos em todas as } \\
\text { variáveis avaliadas quando comparado ao grupo submetido a exercícios de fortalecimento } \\
\text { superficial, avaliado por biofeedback de pressão. }\end{array}$ \\
\hline Ewert et al., 2009 & Não houve diferença significativa entre os grupos. \\
\hline $\begin{array}{l}\text { Carpes, Reinehr e Mota, } \\
2008\end{array}$ & $\begin{array}{l}\text { Os resultados indicaram ausência ou redução da dor lombar, bem como aumento da estabilização } \\
\text { e força do complexo lombar pélvico comparando o pré e pós-treino. }\end{array}$ \\
\hline
\end{tabular}




\begin{tabular}{l|l}
\hline Stevens et al., 2007 & $\begin{array}{l}\text { Resultados indicam que a via de recrutamento muscular pode ser modificada através de um } \\
\text { programa de treino que foca o controle neuromuscular. }\end{array}$ \\
\hline $\begin{array}{l}\text { Fritz, Whitman e Childs, } \\
2005\end{array}$ & $\begin{array}{l}\text { Os indivíduos com hipomobilidade apresentaram benefícios com o programa de manipulação } \\
\text { e exercícios gerais, enquanto que os indivíduos com hipermobilidade se beneficiaram com o } \\
\text { programa de exercícios de estabilização central. }\end{array}$ \\
\hline Hicks et al., 2005 & $\begin{array}{l}\text { A resposta dos pacientes com lombalgia pode ser predita por variáveis clínicas que podem ser } \\
\text { usadas para determinar se ocorrerá benefícios com o programa de exercícios de estabilização } \\
\text { central. }\end{array}$ \\
\hline $\begin{array}{l}\text { Koumantakis, Watson e } \\
\text { Oldham, 2005 }\end{array}$ & $\begin{array}{l}\text { Os exercícios gerais foram mais efetivos a curto prazo comparado aos exercícios de estabilização } \\
\text { central. Os exercícios de estabilização central não promovem benefícios adicionais para } \\
\text { pacientes com dor subaguda ou crônica. }\end{array}$ \\
\hline $\begin{array}{l}\text { Rasmussen-Barr, } \\
\text { Nilsson-Wikmarn e } \\
\text { Arvidsson, 2003 }\end{array}$ & $\begin{array}{l}\text { Não houve diferenças entre os grupos, a curto prazo, nas variáveis avaliadas. A longo prazo, } \\
\text { no entanto, o grupo submetido aos exercícios de estabilização central apresentou melhores } \\
\text { resultados comparado ao grupo submetido a terapia manual. Observou-se que a melhora obtida } \\
\text { necessita de menores períodos de tratamento recorrentes. }\end{array}$ \\
\hline
\end{tabular}

Os autores Ahmed, Rehman e Sibtain (2014), compararam técnicas de mobilização lombar específica e exercícios de estabilização central, e somente exercícios mobilização lombar específica. O grupo que recebeu as duas técnicas apresentou melhor resultado na redução da dor e na instabilidade física.

Resultados semelhantes foram obtidos por Fritz, Whitman e Childs (2005), nos quais os indivíduos que receberam técnicas combinadas de manipulação e exercícios de estabilização central alcançaram melhores resultados, principalmente quando foi constada a presença de hipomobilidade. Assim, os autores concluíram que neste caso, a manipulação vertebral antes dos exercícios de estabilização central aumentaria o benefício terapêutico.

Os dados de Rasmussen-Barr, Nilsson-Wikmarn e Arvidsson (2003) não corroboram os autores anteriores, pois nenhuma diferenças entre os grupos foi encontrada a curto prazo. Entretanto, os autores destacam benefícios a longo prazo nos pacientes submetido aos exercícios de estabilização central comparado as técnicas manipulativas.

Já outro estudo desenvolvido por Koumantakis, Watson e Oldham (2005) constatou que os exercícios de estabilização não parecem trazer benefícios adicionais para pacientes com dor lombar subaguda ou crônica que não possuam sinais clínicos de instabilidade da coluna vertebral.

A eficácia clínica da terapia manual para dor lombar já foi pesquisada em diversos estudos clínicos, controlados e randomizados. Shekelle et al. (1992) em uma revisão sistematizada concluiu que a terapia manual acelera a recuperação de um episódio agudo de dor lombar e os pacientes apresentam $34 \%$ de melhora comparada com outros tratamentos conservadores. Um estudo clínico randomizado e controlado realizado por Bronfor ${ }^{53}$ concluiu que a terapia manual realizada em pacientes com lombalgia aguda ou crônica produz benefícios moderados comparados aos grupos placebo, cuidados médicos e eletroterapia. Resultado semelhante foi descrito em uma revisão sistematizada publicada por Van Tulder, Koes e Bouter (1997), no qual os autores concluíram que a terapia manual realizada em pacientes com dor lombar crônica possui grande eficácia quando comparado ao grupo placebo, entretanto quando comparado ao grupo cuidados médicos, repouso, analgésicos e massagem produziu efeitos moderados.
Desta forma, podemos concluir com base nos dados dos artigos analisados nesta revisão e com base na literatura que a associação de terapias manipulativas com exercícios de estabilização central promove efeitos benéficos aos pacientes com lombalgia.

Além das técnicas manipulativas, destacam-se ainda, os exercícios gerais combinados ou não aos exercícios de estabilização central. Ferreira et al. (2010) utilizaram exercícios de controle motor, exercícios gerais e terapia manipulativa. A técnica de controle motor apresentou melhora no recrutamento do transverso abdominal. O efeito dos exercícios de controle motor sobre a redução da dor superou os participantes que apresentavam pouco recrutamento do transverso abdominal.

Vários estudos tentam responder questionamentos se a instrução dada ao paciente para a realização dos exercícios é capaz de promover melhoras representativas. O protocolo estabelecido por Aluko, DeSouza e Peacock (2013) englobou exercícios clássicos de core e exercícios de fortalecimento global com o mínimo de instrução e comparou a um grupo com o mesmo protocolo, entretanto, os sujeitos neste caso, recebiam instruções adicionais sobre oito músculos de estabilização específicos incluindo o transverso abdominal e os multífidos. Não foram encontradas diferenças entre os grupos.

Unsgaard-Tondel et al. (2010), também estudaram os efeitos da instrução individual na realização dos exercícios de controle motor guiados por ultrassom. Assim como Aluko, DeSouza e Peacock (2013), também não foram encontradas diferenças significativas entre os grupos no que se refere a dor ou qualquer outra medida avaliada no pós-tratamento imediato. Entretanto, após um ano, foram observadas diferenças entre os grupos, sendo que os sujeitos que realizaram os exercícios instruídos apresentaram melhores respostas na redução da dor.

Saner et al. (2011), em um estudo ainda não finalizado, buscam responder os questionamentos sobre os efeitos da instrução na realização dos exercícios para controlar movimentos específicos e exercícios gerais visando melhorar a flexibilidade e endurance.

Kumar (2011) comprovou eficácia da estabilização central em seu estudo comparado a um grupo placebo. A técnica de estabilização central também foi superior a exercícios 
de fortalecimento gerais, conforme demonstrado na pesquisa de França et al. (2010).

Ao comparar os efeitos dos exercícios de estabilização central com a fisioterapia convencional, observou-se que a estabilização central é superior quando são avaliados a força e o controle postural (MUTHUKRISHNAN et al., 2010).

França et al. (2012), verificaram que os exercícios focando isometria do músculo eretor da espinha e o tríceps sural não são efetivos na ativação do músculo transverso abdominal ao contrário dos exercícios de estabilização segmentar.

Pesquisas recentes apresentam os músculos: transverso do abdome e multífido como os principais estabilizadores intrínsecos (VOLPATO et al., 2012). Estes músculos profundos possuem inserções segmentares na coluna lombar e são, portanto, capazes de prover controle e rigidez a cada segmento (KISNER; KOLBY, 2009). As fibras profundas dos músculos multífidos e transverso do abdome são as primeiras a se tornarem ativas quando há um distúrbio postural decorrentes de movimentos rápidos dos membros (KISNER; KOLBY, 2009).

O grupo muscular multífido multifacetado tem uma alta distribuição de fibras do tipo 1 e uma grande rede capilar, o que enfatiza seu papel de estabilizador tônico. Suas inserções segmentares são capazes de controlar o movimento dos segmentos da coluna, assim como aumentar sua rigidez. Os músculos multífido e eretor da coluna ficam encapsulados pelas camadas posterior e média da fáscia lombodorsal, de modo que a massa muscular e a contração aumentam a tensão da fáscia, aumentando sua função estabilizadora (KISNER; KOLBY, 2009).

Hicks et al. (2005), realizaram um programa de exercícios de estabilização padronizado. Este programa foi projetado para estimular padrões motores de estabilização para os músculos primários da coluna vertebral, incluindo o reto abdominal, transverso abdominal, oblíquos internos, eretor da espinha, multífido e quadrado lombar. De acordo com os autores, os resultados deste estudo precisam se replicados e analisados em um ensaio clínico randomizado controlado antes de ser recomendado para uso generalizado.

Desta forma, a presente revisão sistematizada permitiu constatar, em vários trabalhos, a eficácia da estabilização segmentar nas lombalgias e, principalmente, na prevenção de sua recidiva. Entretanto, outros estudos demonstram que o assunto ainda não está totalmente esclarecido, sendo necessárias outras formas de avaliação que permitam atribuir os benefícios alcançados às técnicas executadas.

\section{Considerações finais}

A literatura analisada sugere que os exercícios de estabilização são positivos no tratamento de pacientes com disfunção da coluna lombar crônica de origem não específica, mostrando ser eficaz também na melhora da instabilidade.

De acordo com os dados analisados, estes exercícios parecem ser mais efetivos quando associados as outras intervenções, tais como terapias manipulativas e exercícios gerais.

Porém, os artigos analisados não deixam claro se os exercícios de estabilização central são superiores às técnicas de terapia manual ou à tratamentos convencionais como os exercícios gerais.

Os artigos analisados basearam seus resultados principalmente na resposta subjetiva de questionários, escalas e inventários dos participantes. Desta forma, fazem-se necessárias formas de avaliação que realmente evidenciem os benefícios dos exercícios de estabilização central de forma menos subjetiva.

Apesar de a pesquisa ter sido realizada no intervalo de dez anos, observa-se que não houve evolução nas metodologias utilizadas para avaliar os benefícios dos exercícios de estabilização central. Assim conclui-se que serão necessários avanços principalmente no que se refere a metodologia empregada nos trabalhos científicos.

\section{Referências}

AHMED, R.; SHAKIL-UR-REHMAN, S.; SIBTAIN, F. Comparison between specific lumber mobilization and core-stability exercises with core-stability exercises alone in mechanical low back pain. Pak J Med Sci., v. 30, n. 1, p. 157-160, 2014.

ALUKO, A.; De SOUZA, L.; PEACOCK, J. The effect of core stability exercises on variations in acceleration of trunk movement, pain, and disability during an episode of acute nonspecific low back pain: a pilot clinical trial. Journal of Manipulative and Aluko et al Physiological Therapeutics., v. 36, n. x, p. 497-504, 2013.

ANDERSON-PEACOCK, E. et al. Chiropratic clinical practice guideline: evidence based treatment of adult neck pain not due to whiplash. J Can Chiropr Assoc., v. 49, n. 3, p. 158-209, 2005.

BRONFORT, G. Spinal manipulation: current state of research and its indications. Neurol. Clin., v. 17, n. 1, p. 91-111, 1999.

CARPES, F.P; REINEHR, F.B.; MOTA C.B. Effects of a program for trunk strength and stability on pain, low back and pelvis kinematics, and body balance: A pilot study.

Journal of Bodywork and Movement Therapies, v. 12, p. 22-30, 2008.

CHOWN, M. et al. A prospective study of patients with chronic back pain randomised to, group exercise, physiotherapy or osteopathy. Physiotherapy, v. 94, n. 1, p. 21-28, 2008.

COLLINS, N.; TEYS, P.; VICENZINO, B. The initial effect of a mulligan's mobilization with movement technique on dorsiflexion and pain in subacute ankle sprains. Man Ther., v. 9, n. 2, p. 77-82, 2004.

DE VITTA, A. A lombalgia e suas relações com o tipo de ocupação, com a idade e o sexo. Rev Bras Fisioter., v. 1, p. 67-72, 1996.

DEYO, R. A. et al. Cost, controversy, crisis: low-back pain and the health of the public. Annu. Rev. Public. Health., v. 12, p. 141-156, 1991. 
EWERT, T. et al. The Comparative Effectiveness of a Multimodal Program Versus Exercise Alone for the Secondary Prevention of Chronic Low Back Pain and Disability. American Academy of Physical Medicine and Rehabilitation., v. 1, p. 798-808, 2009.

FAIRBANK, J. C. T.; PYNSENT, P. B. The Oswestry Disability Index. Spine. V. 25, p. 2940-53, 2006.

FERREIRA, P. H. et al. Changes in recruitment of transversus abdominis correlate with disability in people with chronic low back pain. Br J Sports Méd., v. 44, p. 1166-1172, 2010.

FRANÇA, F. J. R. et al. Estabilização segmentar da coluna lombar nas lombalgias: uma revisão bibliográfica e um programa de exercícios. Fisioterapia e Pesquisa, v. 15, n. 2, p. 200-206, 2008.

FRANÇA, F. R. et al. Effects of muscular stretching and segmental stabilization on functional disability and pain in patients with chronic low back pain: A randomized, controlled trial. Journal of Manipulative and Aluko et al Physiological Therapeutics., v. 35, n. 4, p. 279-285, 2012.

FRANÇA, F. R. et al. Segmental stabilization and muscular strengthening in chronic low back pain - a comparative study. Clinics., v. 65, n. 10, p. 1013-1017, 2010.

FRITZ, J. M.; WHITMAN, J. M.; CHILDS, J. D. Lumbar Spine Segmental Mobility Assessment: An Examination of Validity for Determining Intervention Strategies in Patients With Low Back Pain. Arch Phys Med Rehabil., v. 86, p. 1745-1752, 2005.

GIFT, A. G. Visual analogue scales: measurement of subjective phenomena. Nurs Res., v. 38, p. 286-288, 1989.

GIL, A. C. Métodos e técnicas de pesquisa Social. 5 ed. São Paulo: Atlas, 1999.

GRIFFIN, D.; HARMON, D.; KENNEDY, N. Do patients with chronic low back pain have an altered level and/or pattern of physical activity compared to healthy individuals? A systematic review of the literature. Physiotherapy, v. 98, p. 13-23, 2012.

HALL, T. et al. Efficacy of a C1-C2 self-sustained natural apophyseal glide (SNAG) in the management of cervicogenic headache. J Orthop Sports Phys Ther., v. 37, n. 3, p. 100-1007, 2007.

HEBERT, J. J. et al. The relationship of transversus abdominis and lumbar multifidus activation and prognostic factors for clinical success with a stabilization exercise program: a cross-sectional study. Arch. Phys. Méd. Rehabil., v. 91, p. 78-85, 2010.

HICKS, G.E. et al. Preliminary Development of a Clinical Prediction Rule for Determining Which Patients With Low Back Pain Will Respond to a Stabilization Exercise
Program. Arch Phys Med Rehabil., v. 86, p. 1753-1762, 2005.

\section{KALTENBORN, F. M. Mobilização Manual das}

Articulações. Vol. I. São Paulo:Manole, 2001.

KISNER, C.; KOLBY, L. A. Exercícios Terapêuticos: Fundamentos e Técnicas. 5. ed. Barueri, SP, 2009.

KOUMANTAKIS, G. A.; WATSON, P. J.; OLDHAM, J. A. Trunk Muscle Stabilization Training Plus General Exercise Versus General Exercise Only: Randomized Controlled Trial of Patients With Recurrent Low Back Pain. Phys Ther., v. 85, p. 209-225, 2005.

KRISMER, M.; VAN TULDER, M. Low back pain (nonspecific). Best Practice \& Res Clin Rheumat., v.21, n.1, p. 77-91, 2007.

KUMAR, S. P. Efficacy of segmental stabilization exercise for lumbar segmental instability in patients with mechanical low back pain: A randomized placebo controled crossover study. N Am J Med Sci., v. 3, n. 10, p. 456-461, 2011.

LAST, J.M. Making the dictionary of epidemiology. Int J Epidemiol., v. 25, n. 5, p. 1098-1101, 1996.

MacFARLANE, T.V. Sample size determination for research projects. J Orthod., v. 30, n. 2, p. 99-100, 2003.

MAITLAND, G. et al. Maitland Manipulação vertebral. 7. ed. Rio de Janeiro: Elsevier, 2007.

MARCONDES, F. B.; LODOVICHI, S. S.; CERA, M. Terapia manipulativa ortopédica na dor vertebral crônica: uma revisão sistemática. Acta Fisiatr., v. 17, n. 4, p. 180-187, 2010.

MELZACK, R. The short-form McGill Pain Questionnaire. Pain., v. 30, n. 2, p. 191-197, 1987.

MONTEIRO, J. et al. Questionário de incapacidade de Roland Morris, Adaptação e Validação para os doentes de língua portuguesa com lombalgia. Acta Méd Port., v. 23, p. 761-766, 2010.

MUTHUKRISHNAN, R. et al. The differential effects of core stabilization exercise regime and conventional physiotherapy regime on postural control parameters during perturbation in patients with movement and control impairment chronic low back pain. Sports Medicine, Arthroscopy, Rehabilitation, Therapy \& Technology. v. 2, p. 13-26, 2010.

NORMANDO, D.; ALMEIDA, M. A. O.; QUINTÃO, C. C. A. Análise do emprego do cálculo amostral e do erro do método em pesquisas científicas publicadas na literatura ortodôntica nacional e internacional. Dental Press J Orthod., v. 16, n. 6, p. 1-9, 2011.

NUNES-JUNIOR, P.C.; MONNERAT, E. Comparação 
dos tratamentos conservador, cirúrgico e através da mobilização neural no tratamento da hérnia de disco lombar. Fisioterapia Brasil, v. 13, n. 2, p. 155-161, 2012.

PANJABI, M. M. Clinical spinal instability and low back pain. J. Electromyogr. Kinesiol., v. 13, n.4, p. 371-379, 2003.

PAVIN, L. N.; GONÇALVES, C. Principles of core stability in the training and in the rehabilitation: review of literature. J. Health Sci. Inst., v. 28, n. 1, p. 53-55, 2010.

RASMUSSEN-BARR, E.; NILSSON-WIKMAR, L.; ARVIDSSON, I. Stabilizing training compared with manual treatment in sub-acute and chronic low-back pain. Manual Therapy., v. 8, n. 4, p. 233-241, 2003.

ROLAND, M.; MORRIS, R. A. study of natural history of low back pain. Part I: development of reliable and sensitive measure of disability in low back pain. Spine. v. 8, p. 141144, 1983.

ROUWER, S. et al. Reliability and stability of the Roland Morris Disability Questionnaire: intra class correlation and limits of agreement. Disabil Rehabil., v. 26, p. 162-165, 2004.

SANER, J. et al. Movement control exercise versus general exercise to reduce disability in patients with low back pain and movement control impairment. A randomised controlled trial. BMC Musculoskeletal Disorders, v. 12, p. 207- 215, 2011 .

SANTOS, C.C. et al. Aplicação da versão brasileira do questionário de dor Mcgill em idosos com dor crônica. Acta Fisiatr., v. 13, n. 2, p. 75-82, 2006.

SHEKELLE, P. et al. Spinal manipulation for low-back pain. Annals of Internal Medicine, v. 117, n. 7, p. 590$598,1992$.

STANDAERT, C. J.; WEINSTEIN, S. M.; RUMPELTES, J. Evidence-informed management of chronic low back pain with lumbar stabilization exercises. Spine J., v. 8, p. 114$20,2008$.

STANMORE, T. Pilates para as Costas. Barueri, SP: Manole, 2008.

STEVENS V. K. et al. The influence of specific training on trunk muscle recruitment patterns in healthy subjects during stabilization exercises. Manual Therapy., v. 12, p. 271-279, 2007.

UNSGAARD-TONDEL, M. et al. Motor Control Exercises, Sling exercises, and General Exercises for Patients With Chronic Low Back Pain: A Randomized Controlled Trial With 1-Year Follow-up. Phys Ther., v. 90, n. 10, p. 14261440, 2010.

VAN TULDER, N. W.; KOES, B. W.; BOUTER, L. M.
Conservative treatment of acute and chronic nonspecific low back pain: a systematic review of randomized controlled trials of the most common intervations. Spine, v. 22, n. 18, p. 2128-2156, 1997.

VICENZINO, B.; COLLINS, D.; WRIGHT, A. Na investigation of the interrelationship between manipulative therapy-induced hypoalgesia and sympathoexcitation.

Journal Manipulative and Physiological Therapeutics, v. 21, n. 7, p. 448-453, 1998.

VITTA, A. A Lombalgia e suas Relações com o Tipo de Ocupação, com a Idade e o Sexo. Rev. Bras. Fisiot., v. I, n. 2, p. 67-72, 1996.

VOLPATO, C.P. et al. Exercícios de estabilização segmentar lombar na lombalgia: revisão sistemática da literatura. Arq. Méd. Hosp. Fac. Cienc. Med. Santa Casa São Paulo, v.57, n. 1, p. 35-40, 2012.

WADDELL, G. et al. A Fear-Avoidance Beliefs Questionnaire (FABQ) and the role of fear-avoidance beliefs in chronic low back pain and disability. Pain. v. 52, p. 157-168, 1993.

WILLARDSON, J. M.; FONTANA, F. E.; BRESSEL, E. Effect of surface stability on core muscle activity for dynamic resistance exercises. Int J Sports Physiol Perform., v.4, p. 97-109, 2009.

WILSON, E. The Mulligan concept: NAGS, SNAGS and mobilizations with movement. Journal of Bodywork and Movement Therapies, v. 5, n. 2, p. 81-88, 2001.

Recebido em: 19/07/2017 Aceito em: 19/02/2018 